\title{
Erweiterung des Emissionshandels löst aktuelles Klimaschutzproblem nicht
}

\begin{abstract}
Um die energie- und klimapolitischen Ziele der Bundesregierung zu erreichen, müssen fossile Heiz- und Kraftstoffe stärker bepreist werden. Welche unterschiedlichen Optionen für eine $\mathrm{CO}_{2}$-Bepreisung existieren? Bei ihrer Bewertung spielen neben ökonomischen Argumenten auch die juristische und politische Durchsetzbarkeit eine Rolle. Von Claudia Kemfert, Sophie Schmalz und Nicole Wägner
\end{abstract}

\section{Die Notwendigkeit einer $\mathrm{CO}_{2}$-Bepreisung}

Die Sektoren Wärme und Verkehr leisten im Gegensatz zum Stromsektor bislang nur einen geringen Beitrag zum Klimaschutz: Gemessen an den Emissionsniveaus von 1990 und 2014 trägt die Energiewirtschaft mit 108 Millionen Tonnen $\mathrm{CO}_{2}$-Äquivalenten absolut am meisten zur Emissionsreduktion bei [1]. Mit dem Klimaschutzplan 2050 beschloss die Bundesregierung im Jahr 2016 sektorspezifische Ziele zur Emissionsreduktion für die Bereiche Gebäude und Verkehr, die bis zum Jahr 2030 erreicht werden sollen. Demnach soll im Gebäudebereich eine Minderung um 66 bis 67\% gegenüber dem Emissionsniveau von 1990 erreicht werden. Die Treibhausgasemissionen des Verkehrs müssen im gleichen Zeitraum um 40 bis $42 \%$ sinken um die Sektorziele 2030 zu erreichen (BMU 2016). Vor dem Hintergrund der bisherigen und bislang auch künftig zu erwartenden Entwicklungen der Emissionen sowie der derzeitigen Bandbreite an Maßnahmen, scheint eine Zielerreichung für die beiden Sektoren unwahrscheinlich (SRU 2017).

\section{Mögliche Ausweitung des Emissions- handels}

\subsection{Aktuelle Debatte und Vorschläge für eine}

\section{Ausweitung}

Seit einigen Jahren wird diskutiert, weitere Sektoren, wie den Verkehr und die Wärme, in den Emissionshandel einzubeziehen. Um zu bewerten, ob der Emissionshandel hierfür ein geeignetes Instrument darstellt, sollte nicht nur hinsichtlich der Sektoren (Verkehr und Wärme) und der politischen Ebene (national bis europaweit) sondern auch zwischen kurzund langfristigen Optionen differenziert werden.
Derzeit werden insbesondere zwei Optionen, weitere Sektoren über den Zertifikatehandel zu bepreisen, diskutiert: Die erste Möglichkeit ist die langfristige Erweiterung des EU-Emissionshandels (EU-ETS) auf zusätzliche Sektoren auf EU-Ebene. Dies ist für alle oder eine Gruppe an Mitgliedstaaten denkbar. Die zweite Möglichkeit wäre, ein auch kurzfristig umsetzbares separates System für den Emissionshandel im Verkehrs- und Wärmesektor einzuführen - auf nationaler Ebene oder im Rahmen einer „Koalition der Willigen“, also Mitgliedstaaten, die analog nationale Handelssysteme für die Sektoren etablieren (siehe Absatz Politische Durchsetzbarkeit).

Im Rahmen einer möglichen Ausweitung des Emissionshandels auf den Wärme- und Verkehrssektor wird meist der sogenannte Upstream-Ansatz als vorzugswürdige Umsetzungsoption betrachtet (Deuber 2002; Flachsland et al. 2011; Kampman et al. 2008; Karplus et al. 2013; UBA 2014). Mit Upstream werden im engeren Sinne Produzenten und Importeure von Energieträgern bezeichnet; im weiteren Sinne auch weitere Verarbeitungsstufen und der Transport, wie die Raffinerien oder Pipelinesysteme (UBA 2014). Der Downstream-Ansatz sieht hingegen vor, dass die Endverbraucher der Energieträger sich dem Emissionshandel anschließen und so zertifikatepflichtig werden. Aufgrund der Vielzahl an zertifikatepflichtigen Verbrauchern würde dies für die Sektoren Wärme und Verkehr enorme Komplexitäten in der Umsetzung mit sich bringen. Mit Hybrid-Systemen können beide Ansätze miteinander verbunden werden, indem große Anlagen weiterhin durch den Downstream-Emissionshandel erfasst werden und Sektoren mit einem größeren Anteil an kleineren Emittenten, wie in den Sektoren Wärme und Verkehr, einem Upstream-System unterliegen (UBA 2014).

\section{2 Ökonomische Aspekte}

Trotz der theoretisch hohen Effizienz sprechen gegenwärtig verschiedene Argumente gegen die Ausweitung des Emissionshandels auf den Verkehrssektor - insbesondere als primäres oder gar alleiniges Instrument zur Treibhausgasreduktion (Holm-Müller et al. 2010). So leidet die in der Theorie hohe Effizienz ebenso wie die Treffsicherheit, also die ökologische Effektivität des Instruments, in der Praxis unter Umsetzungsdefiziten (Cambridge Econometrics 2014; Elmer 2016; Mock et al. 2014; Kasten et al. 2015). Die optimale Festlegung der Höhe des Emissionsminderungsziels (Cap) wird aufgrund unvollständiger Information erschwert, was wiederum zu Unsicherheiten über den sich bildenden $\mathrm{CO}_{2}$-Preis führt. Die politische Durchsetzungsfähigkeit ist nicht ausreichend vorhanden und 
die Cap-Höhe wird zu niedrig gewählt, Ausnahmen für die Industrie (kostenlose Vergabe der Zertifikate) wegen angeblicher Carbon-Leakage-Gefahr, also der Sorge vor Verlagerung industrieller Produktion und Emissionen, gewährt. Aufgrund dieser ist bei den derzeitigen und auch bis 2030 erwartbaren Zertifikatepreisen nur mit geringen ökonomischen wie ökologischen Lenkungswirkungen zu rechnen.

Bei einer Erweiterung des Emissionshandels auf den Wärmesektor ist von zentraler Bedeutung, dass der Zertifikatehandel gegenüber einer Besteuerung eine höhere (insbesondere langfristige) Preisunsicherheit mit sich bringen kann. Dieses Problem besteht grundsätzlich für alle Sektoren, wobei vor allem im Gebäudebereich langfristige Preissignale notwendig sind, um Anreize für energetische Sanierungen zu setzen. Eine Besteuerung mit planbaren Preispfaden scheint daher sinnvoller. Auch für den Wärmesektor kann die Bepreisung im Rahmen des Emissionshandels ein Baustein sein, damit Gebäude stärker energetisch saniert werden und die Wärmebereitstellung aus erneuerbaren Energien langfristig die günstigere Alternative darstellt. Allerdings besteht hierbei ein Mieter-Vermieter-Dilemma. Das Preissignal beim Upstream-Emissionshandel betrifft die Mieter/innen, da diese für höhere Heizkosten aufkommen müssen. Die Investitionskosten der Sanierung sind jedoch von Seite der Vermieter/innen zu tragen (Hermann et al. 2014).

Die $\mathrm{CO}_{2}$-Vermeidungskosten für die beiden großen NichtETS-Sektoren Verkehr und Gebäude liegen größtenteils über denen des Energiesektors. Daher dürfte die Einbeziehung der Sektoren in den EU-ETS eine Dekarbonisierung des Energiesektors zunächst beschleunigen, anstatt nennenswerte Emissionsminderungen in den beiden Sektoren, insbesondere dem Verkehrssektor, zu bewirken (Agora Energiewende et al. 2018).

Unter Zugrundelegung der derzeitigen Zertifikatspreise würde der Verkehrssektor bei einer Einbindung in den Emissionshandel seine Minderungsverpflichtungen voraussichtlich in hohem Maße durch den Zukauf von Zertifikaten decken. Dieser Zukauf würde jedoch nicht zu zusätzlichen Emissionsminderungen in gleicher Höhe in anderen Sektoren führen, sondern stattdessen den derzeit im Emissionshandel bestehenden hohen Zertifikateüberschuss senken. Diese überschüssigen Zertifikate würden andernfalls zu großen Teilen in die neu geschaffene Marktstabilitätsreserve überführt. Hieraus könnten sie - gemäß derzeit diskutierter Reformvorschläge für den Emissionshandel - zu einem späteren Zeitpunkt endgültig stillgelegt werden (Cowart et al. 2017; Matthes 2017) und somit zu einer Verringerung der Emissionen führen. Ein Zukauf (ansonsten überschüssiger) $\mathrm{CO}_{2}$-Zertifikate durch den Verkehrssektor anstelle eigener Emissionsminderungen würde folglich die möglichen Emissionsminderungen der EU reduzieren. Es wäre somit klimapolitisch wirksamer, wenn der Verkehrssektor seine Klimaziele durch eigene Anstrengungen und Maßnahmen, unter anderem durch separate Preissignale für den Energieverbrauch und die $\mathrm{CO}_{2}$-Emissionen, erreichte.
Auch im Falle eines durch eine erhebliche Zertifikateknappheit gekennzeichneten Emissionshandels könnte durch die Einbeziehung des Verkehrssektors ein klimapolitisch problematisches Szenario eintreten. In einer solchen Knappheitssituation könnte die Zertifikatenachfrage des Verkehrs zu einem starken Anstieg der Zertifikatepreise führen. Dieser könnte möglicherweise sogenannte Carbon-Leakage-Effekte in der Industrie nach sich ziehen, wodurch die tatsächliche klimapolitische Effektivität des Emissionshandels geschwächt würde (Naegele et al. 2019; Neuhoff et al. 2015). Bei einer strukturellen Reform des EU-ETS werden daher Carbon-Leakage-Schutzmaßnahmen benötigt, auch um stabile Rahmenbedingungen für umfassende Investitionen hin zu einer klimafreundlicheren Herstellung und Nutzung insbesondere $\mathrm{CO}_{2}$-intensiver Materialien zu ermöglichen (Neuhoff et al. 2015). Zudem könnte der EU-ETS um ein sogenanntes Klimapfand ergänzt werden, das als Abgabe auf die Nutzung emissionsintensiver Grundstoffe erhoben würde. Konsument/innen industrieller Erzeugnisse müssten demnach eine Abgabe zahlen, die sich an der durchschnittlichen $\mathrm{CO}_{2}$-Intensität der für die Herstellung dieser Produkte benötigten Grundstoffe orientiert. Dadurch könnten die $\mathrm{CO}_{2}$-Emissionen stark reduziert werden, ohne dass die Verlagerung der Industrie ins Ausland befürchtet werden müsste. Durch eine pauschale Rückerstattung der Erlöse an die Bürger/innen hätte das Klimapfand ein progressives Element, wodurch ärmere Haushalte überdurchschnittlich profitieren würden (Neuhoff et al. 2019). Der Verkehrssektor ist hingegen wenig anfällig für Carbon-Leakage, sodass hier keine Ausweichreaktionen durch eine ansteigende $\mathrm{CO}_{2}$-Bepreisung zu befürchten sind.

Erst langfristig könnte die Einbeziehung des Verkehrs- und Wärmesektors in den Emissionshandel eine sinnvolle Option sein. Dies gilt gerade mit Blick auf die zunehmende Diversifizierung der Energieträger und seine zunehmende Verschmelzung mit anderen Sektoren, insbesondere dem Stromsektor. Im Zuge einer langfristigen Elektrifizierung des Verkehrs- und Wärmesektors, steigt die Rationalität einer Erweiterung des Emissionshandels (für Wärmesektor siehe z. B. Winkler et al. 2018). So sind derzeit Nutzer/innen von Heizöl einem niedrigeren $\mathrm{CO}_{2}$-Preis ausgesetzt als Verbraucher/innen im Stromsektor (Löschel et al. 2018). Kurzfristig sind hierdurch jedoch nur sehr geringe Impulse für die jetzt einzuleitende Verkehrsund Wärmewende zu erwarten, bei gleichzeitigen Risiken für die klimapolitische Effektivität des Emissionshandels.

\subsection{Juristische Fragen}

Über die rechtliche Umsetzung einer Erweiterung des Emissionshandels im Rahmen von Artikel 24 der Emissionshandelsrichtlinie [2] besteht derzeit Uneinigkeit (Agora Energiewende 2019; BMU 2019; Edenhofer et al. 2019; Nysten 2019; SVR 2019; UBA 2019).

Die Richtlinie definiert Emissionen als "die Freisetzung von Treibhausgasen aus Quellen in einer Anlage“, das heißt im Falle einer Ausweitung des Emissionshandels auf den Wärme- 
und den Verkehrssektor wären die Haushalte und Unternehmen die zertifikatepflichtigen Emittenten. Das Bundesumweltministerium (BMU) vertritt deshalb die Auffassung, dass für eine Einbeziehung der Treibstoff-Produzenten die Emissionshandelsrichtlinie geändert werden müsse, da die Richtlinie auf den Downstream-Ansatz ausgelegt sei (BMU 2019). Auch für eine Erweiterung auf nationaler Ebene, ohne dass diese für alle Mitgliedstaaten gelte, müsste demnach die Emissionshandelsrichtlinie geändert werden. Das würde eine Einigung der Mitgliedstaaten erforderlich machen und wäre damit kurzfristig nicht umsetzbar.

Nysten (2019) kommt ebenfalls zu dem Ergebnis, dass es die Emissionshandelsrichtlinie über Artikel 24 nicht zulässt, den Verkehrs- und Wärmesektor in den EU-ETS mittels Upstream-Emissionen einzubeziehen. Auch ein Downstream-Ansatz ist demnach für den Verkehrsbereich nicht möglich, da der EU-ETS bis auf den Flugverkehr lediglich ortsgebundene Anlagen umfasst. Für den Wärmebereich könnten kleinere Verbrennungsanlagen in einem Downstream-Ansatz einbezogen werden. Für eine nationale Ausweitung auf Upstream-Emissionen im Verkehrs- und Wärmesektor müsste also die Emissionsrichtlinie auf EU-Ebene geändert werden (Nysten 2019).

Der Sachverständigenrat zur Begutachtung der gesamtwirtschaftlichen Entwicklung (SVR) kommt zu dem Ergebnis, dass die Erweiterung des Emissionshandels „zwar rechtlich nicht unumstritten“ (SVR 2019, S. 64), jedoch grundsätzlich wohl durchaus möglich sei, da Artikel 24 der Emissionsrichtlinie eine einseitige Erweiterung des Emissionshandels vorsehe (opt-in). Die Europäische Kommission muss den Antrag eines Mitgliedstaates auf Einbeziehung von Nicht-EU-ETS-Sektoren prüfen, wobei Rat und Europäisches Parlament einer positiven Entscheidung allerdings widersprechen können (SVR 2019; Edenhofer et al. 2019). Demnach können sich laut SVR und Edenhofer et al. (2019) allerdings Widerstände ergeben. Bei Einbeziehung zusätzlicher Sektoren mit höheren Vermeidungskosten dürfte der $\mathrm{CO}_{2}$-Preis steigen, was die kurzfristige Umsetzung erschweren würde (Edenhofer et al. 2019; SVR 2019). Ein Rechtsgutachten im Auftrag der Mittelstands- und Wirtschaftsvereinigung der CDU/CSU (MIT) sowie ein Gutachten im Auftrag der FDP kommen zu dem Ergebnis, dass eine Ausweitung des EU-ETS rechtmäßig sei (Nettesheim 2019; Ohms Rechtsanwälte 2019).

Eine umfassende Prüfung der rechtlichen Umsetzbarkeit ist aufgrund der unterschiedlichen Einschätzungen unumgänglich. Grundsätzlich ist eine Änderung der Emissionsrichtlinie möglich, wobei der Rat mit einer qualifizierten Mehrheit sowie das Europäische Parlament zustimmen müssten (SVR 2019).

\subsection{Politische Durchsetzbarkeit}

Unabhängig von juristischen Fragen würde eine Änderung der Emissionshandelsrichtlinie zur Erweiterung des EU-ETS die Initiative der Europäischen Kommission erforderlich machen. Wie aus einer Befragung der Bundesregierung am 26. Juni 2019 hervorgeht, schätzt Bundeskanzlerin Angela Merkel eine
Erweiterung des EU-ETS auf EU-Ebene für alle Mitgliedstaaten als „nicht zielführend“ ein, weil hierfür einstimmige Entscheidungen erforderlich wären. Sie präferiert ein separates Handelssystem für die Sektoren Wärme und Verkehr - in Zusammenarbeit mit Frankreich und den Niederlanden als „Koalition der Willigen“. Die Konrad-Adenauer-Stiftung (KAS) plädiert hingegen für „ein nationales Emissionshandelssystem für den Verkehrs- und Gebäudebereich als Übergangslösung“, dass in Form eines Upstream-Modells für ein geschlossenes Handelssystem eingeführt werden soll (KAS 2019). Gemäß Beschluss des Klimakabinetts vom 20. September 2019 soll ab 2021 ein nationales Emissionshandelssystem eingeführt werden: Ab 2021 sollen Emissionszertifikate zu einem Festpreis von 10 Euro pro Tonne $\mathrm{CO}_{2}$ ausgegeben werden; der Festpreis soll bis 2025 auf 35 Euro pro Tonne $\mathrm{CO}_{2}$ steigen. Erst im Jahr 2026 soll ein Cap festgelegt und die Preisbildung dem Zertifikatemarkt überlassen werden, begrenzt durch Mindest- und Höchstpreise von 35 beziehungsweise 60 Euro je Tonne $\mathrm{CO}_{2}$.

Kurzfristig scheint eine EU-weite Ausweitung des EU-ETS auf die Sektoren Verkehr und Wärme aufgrund rechtlicher sowie politischer Differenzen kaum umsetzbar. Eine Erweiterung des Emissionshandels auf den Verkehrs- und Wärmesektor ist also nur dann eine Option, wenn Verzögerungen im Klimaschutz in Kauf genommen werden. Zur Erreichung der 2030-Ziele sind allerdings kurzfristige Maßnahmen beziehungsweise Emissionsreduktionen erforderlich. Derzeit liegt der Zertifikatepreis des EU-ETS bei etwa 20 bis 25 Euro pro Tonne $\mathrm{CO}_{2}$. Um nennenswerte Klimaschutzwirkungen in den Sektoren Verkehr und Gebäude zu entfalten, wäre jedoch ein deutlich höherer Preis notwendig, um eine ausreichende Lenkungswirkung zu erzielen (Agora Energiewende 2019). Allerdings wäre mit einer deutlichen Steigerung der Zertifikatepreise die Gefahr von Carbon-Leakage-Effekten verbunden. Darüber hinaus besteht das Risiko, dass im Zuge steigender Zertifikatepreise ein politischer und wirtschaftlicher Druck entsteht, die Preise zu deckeln, was wiederum unzureichende Klimaschutzwirkungen zur Folge hätte. Daher sind im Wärme- und Verkehrssektor weitere flankierende ordnungsrechtliche Instrumente notwendig, um die Sektorziele zu erreichen (Bach et al. 2019a).

\section{Die $\mathrm{CO}_{2}$-Bepreisung durch Reform der Energiebesteuerung}

Eine Alternative zur Einbeziehung der Sektoren Verkehr und Wärme in den Emissionshandel ist es, die Energiebesteuerung $\mathrm{zu}$ reformieren und eine $\mathrm{CO}_{2}$-basierte Komponente in der Energiesteuer einzuführen.

\subsection{Aktuelle Debatte und Vorschläge für eine Ausgestaltung}

Im Rahmen der aktuellen Debatte sieht ein Vorschlag des Deutschen Instituts für Wirtschaftsforschung (DIW) vor, ab dem Jahr 2020 eine $\mathrm{CO}_{2}$-Bepreisung mit einheitlichem Steuersatz von 35 Euro je Tonne $\mathrm{CO}_{2}$ einzuführen, der linear auf 
180 Euro bis zum Jahr 2030 je Tonne $\mathrm{CO}_{2}$ ansteigt (Bach et al. 2019a). Dies stellt eine $\mathrm{CO}_{2}$-orientierte Energiesteuererhöhung dar, die zusätzlich zu bestehenden Steuersätzen eingeführt wird, wobei bestehende Steuersätze gegebenenfalls ebenfalls zu überprüfen sind.

Neben einer reformierten Besteuerung sind für die Erreichung der energie- und klimapolitischen Ziele der Bundesregierung flankierende ordnungsrechtliche Maßnahmen im Wärme- und Verkehrssektor notwendig. Die Lenkungswirkungen, insbesondere einer stärkeren Bepreisung von Heizstoffen, setzen beispielsweise nur begrenzt Anreize für energetische Sanierungen. Maßnahmen wir die Kopplung der Kaltmietaufschläge an tatsächlich erreichte Heizkosteneinsparungen, die Sicherstellung der Qualität von energetischen Sanierungen und die Förderung von Quartierskonzepten könnten hier entgegenwirken (Bach et al. 2019a).

\section{2 Ökonomische Aspekte}

Eine Reform der Besteuerung (Preisinstrument) hat gegenüber dem Emissionshandel (Mengeninstrument) den Vorteil einer höheren Planungssicherheit und Preisstabilität: Im Gegensatz zu den volatilen Preisschwankungen des Emissionshandels, setzt ein festgelegter Preispfad langfristige Preissignale und schafft Anreize für Investitionen in klimafreundlichere Technologien (UBA 2019). Damit schafft das Instrument eine hohe langfristige, dynamische Anreizwirkung. Eine festgelegte und schrittweise Erhöhung der Steuersätze schafft mittel- und langfristig Planungssicherheit für private Haushalte und Unternehmen in ihren Konsum- und Investitionsentscheidungen. Die steigenden Energiepreise sowie das durch die Planungssicherheit reduzierte Preisrisiko machen insbesondere langfristige Klimaschutzinvestitionen attraktiver. Damit schafft das Instrument eine hohe langfristige dynamische Anreizwirkung, welche gegebenenfalls Nachteile bei der statischen Effizienz ausgleichen kann.

Alternativ zu einem ex ante festgelegten Preispfad könnte der Preispfad in Abhängigkeit von der tatsächlich erreichten Emissionsreduktion angepasst werden. Dies würde weniger Planungssicherheit, dafür eine höhere ökologische Treffsicherheit (ökologische Effektivität) bewirken. Die Höhe der Bepreisung würde damit an erzielte ökologische Wirkungen angepasst werden, wie es beispielsweise im Schweizer Bepreisungssystem vorgesehen ist (hier wird der Preispfad als Funktion der Emissionsreduktionen an Zielerreichung gekoppelt, BMU 2019). Dies könnte die Unsicherheit bezüglich der ökologischen Lenkungswirkung mindern.

Unabhängig von der Ausgestaltung sollten die Energieträger entsprechend ihres $\mathrm{CO}_{2}$-Gehalts höher besteuert werden, um längerfristige Preissignale zu senden und notwendige ökonomische Anreize in den Bereichen Wärme und Verkehr zu setzen [3]. Dies kann durch eine Reform der Energiesteuer kurzfristig mit geringem Aufwand umgesetzt werden.

Mit der sinkenden Emissionsintensität in der Stromerzeugung und der zunehmenden Sektorkopplung sollte langfristig zudem die hohe Abgaben- und Umlagenbelastung des Stroms gesenkt werden, beispielsweise durch eine Herabsetzung der Stromsteuer.

Aus ökonomischer Perspektive ist die unterschiedlich starke Belastung der Energieträger ineffizient und führt zu höheren Kosten für den Klimaschutz: Abgaben und Umlagen orientieren sich weder an den mit der Nutzung verbundenen $\mathrm{CO}_{2}$-Emissionen noch am Energiegehalt (Kemfert et al. 2019; SRU 2017; 2019). Dies sollte im Rahmen einer grundlegenden Reformierung der Energiebesteuerung angepasst werden. Steuersätze auf Heiz- und Kraftstoffe müssen steigen und stärker $\mathrm{CO}_{2}$-basiert ausgestaltet werden (Kemfert et al. 2019; Bach et al. 2019b). Im Rahmen einer dynamischen Bepreisung sollte dabei gewährleistet werden, dass die Preissignale im Zuge weiterer Emissionseinsparungen und Effizienzfortschritte ausreichend stark bleiben, um klimapolitische Zielsetzungen zu erreichen. Um den Akteuren ausreichend Planungssicherheit zu bieten, sollte der Anstieg der Preispfade dabei möglichst vorhersehbar gestaltet werden (SRU 2016).

Allerdings kann eine $\mathrm{CO}_{2}$-Bepreisung allein ebenfalls nicht garantieren, dass die Klimaziele bis 2030 erreicht werden: Insbesondere in den Sektoren Gebäude und Verkehr ist die Lenkungswirkung des Preisinstruments zumindest kurzfristig begrenzt, sofern sie nicht in einen komplementären Policy-Mix eingebettet werden. Gründe dafür sind zum einen die niedrige Preiselastizität der Energienachfrage und zum anderen die vorhandenen Marktstrukturen, die Investitionen in $\mathrm{CO}_{2}$-ärmere Technologien hemmen (z. B. Mieter-Vermieter-Dilemma). Daher ist es sinnvoll, die $\mathrm{CO}_{2}$-Besteuerung im Verbund mit anderen Maßnahmen und Instrumenten zu implementieren, um die Sektorziele zu erreichen (Bach et al. 2019a). Zentral sind hierbei Maßnahmen wie Förderprogramme und der Aufbau nachhaltiger Infrastrukturen, damit es den Unternehmen und privaten Haushalten erleichtert wird, auf klimafreundlichere Produktions- und Konsumweisen umzusteigen (UBA 2019). Zudem könnte die $\mathrm{CO}_{2}$-Bepreisung im Gebäudebereich damit gekoppelt werden, dass Kaltmietaufschläge im Einklang mit tatsächlichen Heizkosteneinsparungen, die durch Sanierungen erreicht werden, gebracht werden. Die Sicherstellung der Qualität von energetischen Sanierungen könnte beispielsweise durch contracting-Modelle erhöht werden (Bach et al. 2019a).

Um unerwünschten Verteilungswirkungen entgegen zu wirken, sollten geeignete Maßnahmen dazu beitragen, betroffene Bevölkerungsgruppen nicht zusätzlich zu belasten, wie im Folgenden betrachtet wird.

\subsection{Klimaprämie als soziale Komponente}

Eine Anpassung der Steuern hätte für Haushalte vor allem den Vorteil, dass die Preiserhöhungen transparent und planbar wären. Zudem könnten die Steuereinnahmen als mögliche Kompensationen für einkommensschwache Haushalte genutzt werden. Im Rahmen des EU-ETS bestünde theoretisch ebenso die Möglichkeit, über die Versteigerung der Emissionszertifikate Einnahmen zu generieren, die rückverteilt werden könn- 
ten. Da Zertifikatepreise jedoch naturgemäß schwanken, würden die jährlichen Beiträge stärker schwanken, als wenn das bereits existierende Steuersystem um Einzelkomponenten erweitert werden würde.

Grundsätzlich wirken Energiepreissteigerungen regressiv: Sie belasten Haushalte mit geringerem Einkommen, die ohnehin einen überdurchschnittlich hohen Anteil ihres Einkommens für den Energiebedarf aufwenden müssen, stärker als Haushalte mit hohen Einkommen. Dies gilt unabhängig davon, ob die Energiepreissteigerungen marktgetrieben oder durch politische Steuerungsinstrumente induziert sind. Angesichts dieser Wirkungen müssen Interdependenzen zwischen klimaund sozialpolitischen Zielen in politischen Entscheidungsprozessen berücksichtigt werden, um positive Verteilungswirkungen zu erzielen. Auch für eine Steigerung der gesellschaftlichen Akzeptanz für die Energiewende und den Klimaschutz ist dies förderlich (SRU 2016).

Auch die Ausgaben für Kraftstoffe sind als Anteil des Nettoeinkommens regressiv verteilt: Haushalte mit höherem Einkommen wenden einen geringeren Anteil ihres Einkommens für Kraftstoffe auf als Haushalte mit niedrigerem Einkommen (Bach et al. 2019b). Die absoluten wie anteiligen Ausgaben für Strom und Wärme steigen hingegen bei höheren Einkommen weniger stark, zudem ist ihr Anteil an den Konsumausgaben von Haushalten mit niedrigen Einkommen überdurchschnittlich hoch (SRU 2016). Folglich wirken Preissteigerungen in den Bereichen Strom und Wärme deutlich regressiver als solche für Kraftstoffe. Eine $\mathrm{CO}_{2}$-Bepreisung beziehungsweise Preissteigerungen für Kraftstoffe treffen insbesondere Pendler/innen. Diese könnten durch eine erhöhte Entfernungspauschale [4] oder ein Mobilitätsgeld kompensiert werden (Bach et al. 2019b).

Um private Haushalte, die durch höhere Energiesteuern belastet werden, zu entlasten, wird in der aktuellen öffentlichen Diskussion zunehmend ein einheitlicher Pro-Kopf-Transfer diskutiert. Dies wäre eine hilfreiche Maßnahme, um die Sichtbarkeit der Kompensation und damit die politische wie gesellschaftliche Akzeptanz einer $\mathrm{CO}_{2}$-Bepreisung zu erhöhen. Eine sogenannte „Klimaprämie“ könnte als einheitlicher Betrag, beispielsweise rund 100 Euro, pro Einwohner ausgezahlt werden. Solche Klimabonus-Modelle bestehen bereits in anderen Ländern, insbesondere in der Schweiz (Wissenschaftliche Dienste Deutscher Bundestag 2018). Von einer pro-Kopf-gebundenen Kompensation profitieren Personen und Haushalte, deren Energieverbrauch unterdurchschnittlich ist. Auch Haushalte mit niedrigen Einkommen verbrauchen häufig weniger Energie und würden von einer Pro-Kopf-Kompensation profitieren. $\mathrm{Zu}$ berücksichtigen ist allerdings, dass insbesondere die "Grundsicherung für Arbeitssuchende" nach dem zweiten Buch des Sozialgesetzbuchs (SGB II), oft auch als ALG II oder Hartz IV bezeichnet, finanzielle Zuflüsse regelmäßig mit der Grundsicherung verrechnet. Dies bedeutet, dass die ausgezahlte Kompensation bei Beziehenden von Grundsicherung nicht ankommen würde, obgleich sie Preissteigerungen insbesondere von Strom aus der Grundsicherung aufbringen müss- ten (Gechert et al. 2019). Um der vorgeschlagenen Kompensationslösung zum Erfolg zu verhelfen und damit auch die Akzeptanz einer $\mathrm{CO}_{2}$-Bepreisung zu stärken, ist eine Reform der Grundsicherung für Arbeitssuchende erforderlich. So könnten Überlegungen angestellt werden, die jährliche Rückzahlung nicht auf die Grundsicherung anzurechnen oder aber die Regelsätze über alle Altersgruppen hinweg jährlich um 100 Euro zu erhöhen.

\section{Vergleich der Instrumente und Vorteile einer $\mathrm{CO}_{2}$-Besteuerung}

Die Sektoren Wärme und Verkehr leisten im Gegensatz zum Stromsektor bislang nur einen geringen Beitrag zum Klimaschutz (BMU 2016). Die verschiedenen Energieträger in Deutschland sind unterschiedlich stark mit Abgaben und Umlagen belastet, das Abgabesystem orientiert sich weder konsistent an den mit der Nutzung verbundenen $\mathrm{CO}_{2}$-Emissionen noch am Energiegehalt (SRU 2017; 2019). Damit die energieund klimapolitischen Ziele der Bundesregierung erreicht werden können, müssen die $\mathrm{CO}_{2}$-intensiven Heiz- und Kraftstoffe stärker bepreist werden (Dertinger et al. 2019; Kemfert et al. 2019; SRU 2019). Zentrales Instrument zur $\mathrm{CO}_{2}$-Bepreisung ist gegenwärtig der EU-ETS, der die Sektoren Wärme und Verkehr bislang nicht umfasst. Insbesondere die Erreichung der von der Bundesregierung 2016 beschlossenen Sektorziele für die Bereiche Gebäude und Verkehr bis zum Jahr 2030 (BMU 2016) scheint ohne eine schnellstmögliche verstärkte $\mathrm{CO}_{2}$-Bepreisung unwahrscheinlich (SRU 2017).

Neben der klimapolitischen Notwendigkeit spielen mögliche finanzielle Belastungen durch unzureichenden Klimaschutz eine zunehmende Rolle für stärkere Maßnahmen im Verkehrs- und Gebäudebereich. Deutschland hat sich auf europäischer Ebene verpflichtet, Emissionen, die nicht im Emissionshandel berücksichtigt werden (Nicht-EU-ETS), um 14\% bis 2020 und um 38\% bis 2030 gegenüber 2005 zu reduzieren. Die voraussichtlichen Kosten bei Nichterreichung für den Zeitraum 2018 bis 2020 liegen schätzungsweise bei bis zu 500 Millionen Euro jährlich (EWK 2019).

Über die Ausgestaltung einer verstärkten $\mathrm{CO}_{2}$-Bepreisung für die Sektoren Wärme und Verkehr herrscht in der energiepolitischen Debatte Uneinigkeit. Eine Möglichkeit besteht darin, den EU-ETS auf die bislang nicht abgedeckten Sektoren europaweit, für eine Gruppe von EU-Mitgliedstaaten oder national auszuweiten. Eine weitere Option ist ein separates Emissionshandelssystem für die Sektoren Wärme und Verkehr einzuführen. Eine dritte Option umfasst eine Reform der Besteuerung, indem eine $\mathrm{CO}_{2}$-basierte Komponente in der Energiesteuer eingeführt wird.

Grundsätzlich ist eine $\mathrm{CO}_{2}$-Bepreisung sowohl über den Emissionsrechtehandel als auch über eine reformierte Besteuerung möglich. In der Theorie, also ohne Marktunvollkommenheiten, ohne Marktmacht und bei vollständigen Informationen, führen beide Instrumente zum gewünschten Ergebnis. 
Allerdings gibt es theoretische sowie praktische Unterschiede. Der grundsätzliche Unterschied besteht darin, dass der Emissionshandel mit einem Cap funktioniert und damit ein Instrument der Mengensteuerung ist. Damit gewährt der EU-ETS theoretisch eine hohe Sicherheit, wie viel Emissionen tatsächlich vermieden werden, sprich eine hohe ökologische Treffsicherheit (ökologische Effektivität). Ein Nachteil des Emissionshandels ist dagegen, dass der Preis schwanken kann und das Emissionsminderungsziel ausreichend gewählt werden muss, was bislang politisch nicht ausreichend umgesetzt werden konnte. Eine Besteuerung hat als Preisinstrument hingegen den Vorteil einer höheren Preisstabilität. Dies bedeutet eine höhere Planungssicherheit für besteuerte Einheiten und politische Prozesse sowie eine hohe dynamische ökonomische Effizienz (Innovationswirkung), die insbesondere für die Erreichung langfristiger Klimaschutzziele hilfreich sein kann. Nachteil einer Besteuerung ist hingegen, dass ungewiss ist, wieviel Vermeidung mit einem bestimmten Steuersatz erzielt werden kann, sodass sie über eine geringere ökologische Treffsicherheit verfügt. Diese Unsicherheit kann dadurch vermindert werden, dass die erzielte Vermeidung durch regelmäßige Prüfung der Zielerfüllung und Anpassung der $\mathrm{CO}_{2}$-Steuersätze korrigiert wird (dynamische Bepreisung). Die Planungssicherheit ist besonders wichtig, wenn Emissionen von Personen oder Haushalten besteuert werden. Bei einem stark fluktuierenden ETS-Preis wird dies ungleich schwieriger und müsste gegebenenfalls ständig neu justiert werden. Um den Akteuren ausreichend Planungssicherheit zu bieten, sollte der Anstieg der Preispfade im Rahmen einer Besteuerung möglichst vorhersehbar gestaltet werden (SRU 2016).

Sowohl der Emissionshandel als auch eine reformierte Besteuerung verfolgen das Ziel, $\mathrm{CO}_{2}$ kosteneffizient einzusparen, wobei dem Emissionshandel aus umweltökonomischer Perspektive sowie in der öffentlichen Debatte häufig eine höhere Effizienz zugesprochen wird. Im Rahmen der statischen Effi- zienzanalyse kann mit dem EU-ETS theoretisch kosteneffizient Klimaschutz erreicht werden, indem Emissionen dort eingespart werden, wo es am kostengünstigsten ist. Werden neben der statischen Effizienzanalyse in Bezug auf die Grenzvermeidungskosten jedoch auch die dynamische Effizienz (langfristige Innovationswirkung) sowie die Umsetzungsdefizite betrachtet, lassen sich vor dem Hintergrund bestehender Pfadabhängigkeiten und niedriger Zertifikatepreise Ineffizienzen beobachten (Matthes 2010; Weber et al. 2012). Instrumente mit einer höheren langfristigen Planungssicherheit, wie einer Besteuerung, könnten diese Ineffizienzen begrenzen und Pfadabhängigkeiten beziehungsweise Investitionen, die mit irreversiblen Kosten (sunk costs) in fossile Energien verbunden sind, entgegenwirken.

Eine kurzfristige Umstellung der $\mathrm{CO}_{2}$-Bepreisung durch eine Ausweitung des EU-ETS auf die Sektoren Verkehr und Gebäude scheitert an juristischen Fragen und der politischen Durchsetzbarkeit auf EU-Ebene. Es besteht Uneinigkeit darüber, inwieweit juristische Herausforderungen bestehen, beziehungsweise ob die Emissionshandelsrichtlinie angepasst werden müsste. Nach Auffassung des BMU ist eine Änderung der Emissionshandelsrichtlinie erforderlich - unabhängig davon, ob es sich um eine rein nationale oder eine EU-weite Regelung handeln soll. Eine umfassende Prüfung der rechtlichen Umsetzbarkeit ist aufgrund der unterschiedlichen Einschätzungen unumgänglich. Grundsätzlich ist eine Änderung der Emissionsrichtlinie möglich, wobei der Rat mit einer qualifizierten Mehrheit sowie das Europäische Parlament zustimmen müssten, was Verzögerungen für die Implementierung einer $\mathrm{CO}_{2}$-Bepreisung mit sich bringen würde und die Erreichung der klimapolitischen Sektorziele bis 2030 stark gefährdet.

Die Erweiterung des Emissionshandels ließe sich mit einem Upstream-Ansatz für die Sektoren Verkehr und Gebäude auf EU-Ebene umsetzen; neben den angeführten juristischen Fragen bestehen jedoch auch ökonomische Herausforderungen. Zum einen wäre nur eine überschaubare Anzahl an poten-

\begin{tabular}{|c|c|c|c|}
\hline & & Ausweitung des EU-ETS & $\mathrm{CO}_{2}$-Steuer mit langfristig festgelegtem Preispfad \\
\hline \multirow{3}{*}{$\begin{array}{l}\text { Ökonomische } \\
\text { Effizienz }\end{array}$} & Statistische Kosteneffizienz & Hoch & Mittel \\
\hline & & $\begin{array}{l}\text { Durch Marktmechanismus beim Zertifikatehandel } \\
\text { können Emissionen kosteneffizient eingespart } \\
\text { werden }\end{array}$ & $\begin{array}{l}\text { Unvollständige Informationen über Grenz- } \\
\text { vermeidungskosten bei der Festlegung des Steuer- } \\
\text { satzes }\end{array}$ \\
\hline & $\begin{array}{l}\text { Dynamische Innovations- } \\
\text { wirkung }\end{array}$ & $\begin{array}{l}\text { Mittel } \\
\text { I Langfristige Preisentwicklung schwer antizipierbar }\end{array}$ & $\begin{array}{l}\text { Hoch } \\
\text { I Schafft Planungssicherheit für Investitionsentschei- } \\
\text { dungen der Konsument/innen und Unternehmen }\end{array}$ \\
\hline \multicolumn{2}{|c|}{ Ökologische Treffsicherheit } & $\begin{array}{l}\text { Hoch } \\
\text { Cap muss ausreichend ambitioniert festgelegt } \\
\text { werden }\end{array}$ & $\begin{array}{l}\text { Mittel } \\
\text { Dynamische Bepreisung (in Abhängigkeit von } \\
\text { der tatsächlich erreichten Emissionsminderung) } \\
\text { kann ökologische Treffsicherheit erhöhen }\end{array}$ \\
\hline \multicolumn{2}{|c|}{ Juristische und politische Umsetzbarkeit } & $\begin{array}{l}\text { Problematisch } \\
\text { I Änderung der Emissionshandelsrichtlinie voraus- } \\
\text { sichtlich notwendig } \\
\text { Initiative der Europäischen Kommission und } \\
\text { qualifizierte Mehrheit im Europäischen Rat erfor- } \\
\text { derlich }\end{array}$ & $\begin{array}{l}\text { Mittel } \\
\text { I Änderung des Energiesteuergesetzes erforderlich } \\
\text { Ggf. Änderungen in der Sozialgesetzgebung } \\
\text { notwendig } \\
\text { Entscheidungen auf nationaler Ebene ohne } \\
\text { Beteiligung europäischer Institutionen (kurzfristig } \\
\text { umsetzbar) }\end{array}$ \\
\hline
\end{tabular}

Tabelle: Vor- und Nachteile zweier Alternativen zur $\mathrm{CO}_{2}$-Bepreisung 
ziellen Marktteilnehmenden insbesondere von einer Ausweitung des Handels auf nationaler Ebene oder für eine Gruppe an EU-Mitgliedstaaten betroffen, so zum Beispiel eine geringe Anzahl von Mineralölunternehmen in Deutschland. Dies würde die ökologische Effektivität, also die Treffsicherheit der Minderungsvorhaben, und die ökonomische Effizienz (statische und dynamische Kostenanalyse) des Instruments begrenzen. Ein Downstream-Ansatz wäre hingegen sehr komplex. Des Weiteren ist bei einer Erweiterung des Emissionshandels auf die Sektoren Verkehr und Wärme von zentraler Bedeutung, dass der Zertifikatehandel gegenüber einer Besteuerung eine höhere Preisunsicherheit mit sich bringt. Vor allem im Gebäudebereich sind langfristige Preissignale notwendig, um Anreize für energetische Sanierungen zu setzen. Darüber hinaus sind die Vermeidungskosten insbesondere im Verkehrssektor höher als im Energiesektor. Dies würde zu einem starken Anstieg des $\mathrm{CO}_{2}$-Preises führen, was die kurzfristige Umsetzung erschweren würde (Edenhofer et al. 2019; SVR 2019).

Langfristig kann die Erweiterung des EU-ETS auf europäischer Ebene für alle Mitgliedstaaten eine Option sein, sofern dem Problem des stets zu niedrigen Caps ausreichend begegnet wird. Auch um verlässliche Preissignale für langfristige klimafreundliche Innovationen zu senden, sollte im EU-ETS ein $\mathrm{CO}_{2}$-Mindestpreis eingeführt werden.

Eine verstärkte $\mathrm{CO}_{2}$-Bepreisung aufgrund einer reformierten Besteuerung könnte ab dem Jahr 2020 mit einheitlichem Steuersatz von 35 Euro je Tonne $\mathrm{CO}_{2}$ eingeführt werden, der linear auf 180 Euro bis zum Jahr 2030 je Tonne $\mathrm{CO}_{2}$ steigt (Bach et al. 2019a). Dies wäre gegenüber dem EU-ETS auf nationaler Ebene kurzfristig umsetzbar. Die Gefahr einer Verzögerung von Klimaschutzmaßnahmen aufgrund juristischer Fragen und politischer Durchsetzungsprobleme ist gegenüber einer Ausweitung des EU-ETS oder eines separaten Emissionshandelssystems geringer.

Darüber hinaus sprechen soziale Aspekte für eine Besteuerung und gegen den Emissionshandel, da mögliche finanzielle Kompensationen für einkommensschwache Haushalte leichter und mit geringeren Transaktionskosten umgesetzt werden können. Mit einer Pro-Kopf-Kompensation, der sogenannten „Klimaprämie“, können insbesondere Haushalte mit niedrigem Einkommen profitieren. Neben der sozialpolitischen Notwendigkeit kann dies auch die gesellschaftliche Akzeptanz für eine $\mathrm{CO}_{2}$-Bepreisung steigern. Dabei muss beachtet werden, dass finanzielle Kompensationen nicht mit der „Grundsicherung für Arbeitssuchende" nach dem zweiten Buch des Sozialgesetzbuchs (SGB II) verrechnet werden, damit keine negativen Verteilungswirkungen entstehen, was wiederum Gesetzesänderungen erforderlich machen würde.

Für die Erreichung der energie- und klimapolitischen Ziele der Bundesregierung - insbesondere der Sektorziele bis 2030 sind neben einer reformierten Besteuerung flankierende ordnungsrechtliche Maßnahmen im Wärme- und Verkehrssektor notwendig (Bach et al. 2019a). Für den Verkehrssektor könnten diese Investitionen in klimafreundliche Mobilität, insbe- sondere den öffentlichen Personenverkehr und die Elektromobilität, Förderung des Umweltverbundes, Änderung des Straßenverkehrsrechts und eine streckenabhängige Pkw-Maut umfassen (SRU 2017). Für den Wärmesektor scheint eine komplementäre Förderung von Quartierskonzepten zur Bündelung von Sanierungsmaßnahmen sinnvoll. Auch Konzepte, die auf die Lösung des Mieter-Vermieter-Dilemmas abzielen, wie eine Kopplung der Kaltmietaufschläge an tatsächlich erreichte Heizkosteneinsparungen, kann positive Wirkung entfalten (Bach et al. 2019a).

\section{Anmerkungen}

[1] Relativ zu den Gesamtemissionen entspricht das einer Minderung um $23 \%$ für die Energiewirtschaft. Die prozentuale Minderung im Gebäudebereich war mit $43 \%$ höher, absolut jedoch mit 90 Millionen Tonnen $\mathrm{CO}_{2}$ geringer.

[2] Richtlinie 2003/87/EG des Europäischen Parlaments und des Rates vom 13. 10. 2003 über ein System für den Handel mit Treibhausgasemissionszertifikaten in der Union und zur Änderung der Richtlinie 96/61/EG des Rates, L 275/32 vom 25. 10. 2013, zuletzt geändert durch Richtlinie 2018/410/EU des Europäischen Parlaments und des Rates vom 14. März 2018, L 76/3 vom 19.03. 2018.

[3] Damit die Heiz- und Kraftstoffe entsprechend ihrer tatsächlichen Kosten besteuert werden können, bedarf es grundsätzlich auch eines Abbaus umweltschädlicher Subventionen. So würde der Abbau bestehender Dieselprivilegien zudem Effizienzsteigerungen hervorbringen (für Entlastungen im Verkehrsbereich siehe Bach et al. 2019 a; 2019b).

[4] Die Pendlerpauschale, insbesondere die entfernungsabhängige Pauschale, steht jedoch seit Jahren in der Kritik, falsche sozial- sowie verkehrspolitische Signale zu senden und indirekt als umweltschädliche Subvention zu wirken. Ein Festbetrag würde das Steuersystem vereinfachen und positiv klimapolitische Anreize geben (DIW 2008; Bach 2008; SRU 2012; 2016).

\section{Literatur}

Agora Energiewende/Öko-Institut (Hrsg.) (2018): Vom Wasserbett zur Badewanne. Die Auswirkungen der EU-Emissionshandelsreform 2018 auf $\mathrm{CO}_{2}$-Preis, Kohleausstieg und den Ausbau der Erneuerbaren. Berlin, Agora Energiewende, Öko-Institut

Agora Energiewende (2019): 15 Eckpunkte für das Klimaschutzgesetz. Berlin, Agora Energiewende.

Bach, S. (2008): Die Pendlerpauschale setzt falsche Signale - und zwar unabhängig davon, ob sie beschränkt wird oder nicht. In: DIW Berlin Wochenbericht 16/2008: 212.

Bach, S./Isaak, N./Kemfert, C./Kunert, U./Schill, W.-P./Wägner, N./Zaklan, A./ Schmalz, S. (2019 a): $\mathrm{CO}_{2}$-Bepreisung im Wärme- und Verkehrssektor: Diskussion von Wirkungen und alternativen Entlastungsoptionen. Politikberatung kompakt 140. Berlin, Deutsches Institut für Wirtschaftsforschung.

Bach, S./Kunert, U./Radke, S./Isaak, N. (2019b): $\mathrm{CO}_{2}$-Bepreisung für den Verkehrssektor? Bedeutung und Entwicklung der Kosten räumlicher Mobilität der privaten Haushalte bei ausgewählten verkehrspolitischen Instrumenten. Berlin, Stiftung Arbeit und Umwelt der IG BCE.

BMU (2016): Klimaschutzplan 2050. Klimaschutz-politische Grundsätze und Ziele der Bundesregierung. Berlin, Bundesministerium für Umwelt, Natur und Reaktorsicherheit.

BMU (2019): Warum eine Einbeziehung des Verkehrssektors in den Europäischen Emissionshandel nicht möglich ist. Informationspapier vom 16. September 2019. Berlin, Bundesministerium für Umwelt, Natur und Reaktorsicherheit.

Cambridge Econometrics (2014): The Impact of Including Road Transport in the ETS. A Report for The European Climate Foundation. Cambridge, Cambridge Econometrics. 
Cowart, R./Buck, M./Carp, S. (2017): Aligning Europe's Policies for Carbon, Efficiency, and Renewables: Creating a "Virtuous Cycle" of Performance and Emissions Reduction. Montpelier, Regulatory Assistance Project, Agora Energiewende, Sandbag.

Dertinger, A./Schill, W.-P. (2019): Ansätze zur Umgestaltung von Abgaben und Umlagen bei Heiz- und Kraftstoffen sowie Strom. DIW Roundup 127. Berlin, Deutsches Institut für Wirtschaftsforschung.

Deuber, O. (2002): Einbeziehung des motorisierten Individualverkehrs in ein deutsches $\mathrm{CO}_{2}$-Emissionshandelssystem. Freiburg, Öko-Institut.

DIW Berlin (2008): Pendlerpauschale: Festbetrag soll Steuersystem vereinfachen DIW Berlin: „Klimapolitik sollte Anreize für kurze Arbeitswege setzen“, Pressemitteilung vom 24. Januar 2008. www.diw.de/de/ diw_01.c.78482.de/themen_nachrichten/pendlerpauschale_festbetrag_ soll_steuersystem_vereinfachen_diw_berlin_klimapolitik_sollte_ anreize_fuer_kurze_arbeitswege_setzen.html

Edenhofer, O./Flachsland, C./Kalkuhl, M./Knopf, B./Pahle, M. (2019): Optionen für eine $\mathrm{CO}_{2}$-Preisreform. Berlin, Sachverständigenrat zur Begutachtung der gesamtwirtschaftlichen Entwicklung.

Elmer, C.-F. (2016): The Economics of Vehicle $\mathrm{CO}_{2}$ Emissions Standards and Fuel Economy Regulations: Rationale, Design, and the Electrification Challenge. Berlin, Technische Universität Berlin.

EWK (Hrsg.) (2019): Stellungnahme zum zweiten Fortschrittsbericht der Bundesregierung für das Berichtsjahr 2017. Berlin, Expertenkommission zum Monitoring-Prozess „Energie der Zukunft“.

Flachsland, C./Brunner, S./Edenhofer, O./Creutzig, F. (2011): Climate Policies for Road Transport Revisited (II): Closing the Policy Gap with Cap-andTrade. In: Energy Policy 39/4: 2100-2110.

Gechert, S./Rietzler, K./Schreiber, S./Stein, U. (2019): Wirtschaftliche Instrumente für eine klima- und sozialverträgliche $\mathrm{CO}_{2}$-Bepreisung. Belastungsanalyse. Düsseldorf, Institut für Makroökonomie und Konjunkturforschung.

Holm-Müller, K./Weber, M. (2010): Plädoyer für eine instrumentelle Flankierung des Emissionshandels im Elektrizitätssektor. Berlin, Sachverständigenrat für Umweltfragen.

KAS (Hrsg.) (2019): $\mathrm{CO}_{2}$-Bepreisungsmodelle im Vergleich. Analysen \& Argumente 361. Berlin, Konrad-Adenauer-Stiftung.

Kampman, B./Davidson, M. D./Faber, J. (2008): Emissions Trading and Fuel Efficiency in Road Transport: An Analysis of the Benefits of Combining Instruments. Stockholm, Swedish Environmental Protection Agency.

Karplus, V./Paltsev, S./Babiker, M./Reilly, J. (2013): Should a Vehicle Fuel Economy Standard Be Combined with an Economy-Wide Greenhouse Gas Emissions Constraint? Implications for Energy and Climate Policy in the United States. In: Energy Economics 36: 322-333.

Kasten, P./Schumacher, K./Zimmer, W. (2015): Instrumentenmix im Verkehrssektor: Welche Rolle kann der EU-ETS für den Straßenverkehr spielen? Freiburg, Öko-Institut.

Kemfert, C./Schill, W.-P./Wägner, N./Zaklan, A. (2019): Umweltwirkungen der Ökosteuer begrenzt, $\mathrm{CO}_{2}$-Bepreisung der nächste Schritt. In: DIW Berlin Wochenbericht 13/2019: 215-221.

Löschel, A./Kaltenegger, O. (2018). Nachtrag: Klimaziel 2020 verfehlt: Zeit für eine Neuausrichtung der Klimapolitik. In: ifo Schnelldienst 71/2: 14-17.

Matthes, F. C. (2010): Der Instrumenten-Mix einer ambitionierten Klimapolitik im Spannungsfeld von Emissionshandel und anderen Instrumenten. Freiburg, Öko-Institut.

Matthes, F. C. (2017): Sind komplementäre Maßnahmen zum EU-ETS wirklich nur Nullsummenspiele? Vortrag, Workshop des Öko-Instituts auf den Berliner Energietagen 2017, 05. 05. 2017, Berlin.

Mock, P./Tietge, U./German, J./Bandivadekar, A. (2014): Road Transport in the EU Emissions Trading System: An Engineering Perspective. Washington D. C., International Council on Clean Transportation.

Naegele, H./Zaklan, A. (2019): Does the EU-ETS Cause Carbon Leakage in European Manufacturing? In: Journal of Environmental Economics and Management 2019/93: 125-147.

Nettesheim, M. (2019): Die Einbeziehung des Transportsektors in das Europäische Emissionshandelssystem. Gutachten im Auftrag der Fraktion der Freien Demokraten im Deutschen Bundestag. Tübingen, Universität Tübingen.
Neuhoff, K./Acworth, W./Ismer, R./Sartor, O./Zetterberg, L. (2015): Maßnahmen zum Schutz vor Carbon Leakage für $\mathrm{CO}_{2}$-intensive Materialien im Zeitraum nach 2020. In: DIW Berlin Wochenbericht 29+30/2015: 679-688. Neuhoff, K./Richtstein, J./Zipperer, V. (2019): Klimapfand für eine klimafreundlichere Industrie. In: DIW Berlin Wochenbericht 18/2019: 324-325.

Nysten, J.V. (2019): „Zur Zulässigkeit der Ausweitung des EU-Emissionshandels nach Art. 24 EHS-Richtlinie auf die Bereiche Verkehr und Wärme in Deutschland. Würzburg, Stiftung Umweltenergierecht, Juli 2019.

Ohms Rechtsanwälte (2019): Rechtliche Optionen und Konflikte einer Einbeziehung des Straßenverkehrs in den Emissionshandel. Rechtliche Kurzstellungnahme im Auftrag der Mittelstands- und Wirtschaftsvereinigung der CDU/CSU. Berlin, Ohms Kanzlei für Umwelt-, Energie- und Klimaschutzrecht.

SRU (2012): Umweltgutachten 2012. Verantwortung in einer begrenzten Welt. Berlin, Sachverständigenrat für Umweltfragen.

SRU (2016): Umweltgutachten 2016. Impulse für eine integrative Umweltpolitik. Berlin, Sachverständigenrat für Umweltfragen.

SRU (2017): Umsteuern erforderlich: Klimaschutz im Verkehrssektor. Sondergutachten. Berlin, Sachverständigenrat für Umweltfragen.

SRU (2019): Demokratisch regieren in ökologischen Grenzen - Zur Legitimation von Umweltpolitik. Sondergutachten. Berlin, Sachverständigenrat für Umweltfragen.

SVR (2019): Aufbruch zu einer neuen Klimapolitik. Sondergutachten. Wiesbaden, Sachverständigenrat zur Begutachtung der gesamtwirtschaftlichen Entwicklung.

UBA (2014): Ausweitung des Emissionshandels auf Kleinemittenten im Gebäude- und Verkehrssektor Gestaltung und Konzepte für einen Policy mix. Dessau-Roßlau, Umweltbundesamt.

UBA (2019): $\mathrm{CO}_{2}$-Bepreisung in Deutschland Ein Überblick über die Handlungsoptionen und ihre Vor- und Nachteile. Dessau-Roßlau, Umweltbundesamt.

Weber, M./Hey, C. (2012): Effektive und effiziente Klimapolitik: Instrumentenmix, EEG und Subsidiarität. In: Wirtschaftsdienst 92/13: 43-51.

Wissenschaftliche Dienste Deutscher Bundestag (Hrsg.) (2018): Die $\mathrm{CO}_{2-}$ Abgabe in der Schweiz, Frankreich und Großbritannien. Mögliche Modelle einer $\mathrm{CO}_{2}$-Abgabe für Deutschland. Sachstand WD 8-3000027/18. Berlin, Wissenschaftliche Dienste Deutscher Bundestag.

Winkler, M./Delzeit, R. (2018): Kein „Weiter so“ in der deutschen Klimapolitik: Handlungsvorschläge für die neue Bundesregierung. In: ifw Kiel Policy Briefs 113: 5-13.

\section{AUTORINNEN + KONTAKT}

Dr. Claudia Kemfert leitet seit 2004 die Abteilung Energie, Verkehr, Umwelt am Deutschen Institut für Wirtschaftsforschung (DIW Berlin) und ist seit 2009 Professorin für Energieökonomie und Nachhaltigkeit an der Hertie School of Governance (HSoG). Sophie Schmalz und Nicole Wägner sind Mitarbeiterinnen am DIW.

Deutsches Institut für Wirtschaftsforschung e. V. (DIW Berlin), Mohrenstr. 58, 10117 Berlin. Tel.: +49 30 89789-663, E-Mail: sekretariat-evu@diw.de, Website: https://www.diw.de/deutsch
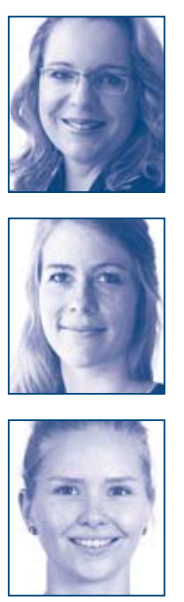